\title{
Ethnicity and sympathetic tone: predictors of the blood pressure response to renal denervation?
}

Yutang Wang

I read with great interest the News \& Views article by Schmieder (How should data from SYMPLICITY HTN-3 be interpreted? Nat. Rev. Cardiol. 11, 375-376; 2014). ${ }^{1}$ Schmieder provided an excellent overview of the randomized, sham-controlled, single-blinded SYMPLICITY HTN-3 trial, ${ }^{2}$ and provided possible explanations why renal denervation did not lower blood pressure in this cohort of patients.

Schmieder stated that several clinical trials on renal denervation "have uniformly demonstrated significant reductions in both systolic and diastolic blood pressure". However, the studies in which renal denervation failed to lower blood pressure were not cited, including four studies that showed no decrease in office blood pressure after renal denervation. ${ }^{3-6}$ An additional seven studies that showed no decrease in 24 -h ambulatory blood pressure after renal denervation were also not mentioned. ${ }^{7-13}$ Admittedly, patient numbers in these 11 trials were small ( $n=6-24)$, and the majority of clinical studies reported have shown a reduction in blood pressure with renal denervation. Nevertheless, being aware of these negative findings is important. ${ }^{3-13}$ For example, Fadl Elmula et al. reported that in six patients with true resistant hypertension, office blood pressure remained unchanged at 1, 3 and 6 months after renal denervation. ${ }^{3}$ This study was the first to use witnessed intake of medication to exclude patients with poor drug adherence. Drug adherence was not verified in the SYMPLICITY HTN trials., 2,14,15

Schmieder offered several explanations for the negative results of the SYMPLICITY HTN-3 trial. One reason suggested was ethnic differences in response to renal denervation. Unlike previous trials, $26.2 \%$ of the study population in the SYMPLICITY HTN-3 trial $^{2}$ were African American. Sub-analyses of ethnicity showed that African-American individuals responded poorly to renal denervation compared with non-African-American individuals, who exhibited "a substantial reduction in mean systolic blood pressure with renal denervation compared with the control group". ${ }^{1}$ However, the difference was no longer significant after adjusting for multiple comparisons. ${ }^{16}$ Schmieder also suggested that the operators of renal denervation in the SYMPLICITY HTN-3 trial lacked experience; however, a previous study demonstrated that renal denervation was ineffective even when performed by very experienced operators. $^{3}$

The 11 trials cited above, ${ }^{3-13}$ together with the SYMPLICITY HTN-3 trial, ${ }^{2}$ clearly demonstrate that renal denervation is not effective for lowering blood pressure in every patient with resistant hypertension, highlighting the need to identify predictors of the blood-pressure response to renal denervation. ${ }^{17}$ A major limitation of the reported clinical trials on renal denervation is that the baseline sympathetic tone of patients has not been investigated. Renal denervation was designed to lower blood pressure by inhibiting the increased sympathetic tone commonly observed in patients with hypertension and, therefore, might not be effective in patients whose sympathetic tone is not increased. ${ }^{18}$ Muscle sympathetic nerve activity, ${ }^{19}$ heart rate variability, ${ }^{20}$ and bloodpressure response to electrical stimulation of renal arteries ${ }^{21}$ might be useful parameters to investigate sympathetic tone. In the future, we must investigate whether increased sympathetic tone predicts the blood-pressure response to renal denervation. If so, this marker will help to identify patients who are likely to benefit from this therapy, and avoid possible harm to nonresponders.

School of Applied and Biomedical Sciences, Federation University Australia, University Drive, Mount Helen, VIC 3350, Australia. yutangwang000@gmail.com

\section{Acknowledgements}

The author is funded by a grant from the National Health and Medical Research Council (1062671).

\section{Competing interests}

The author declares no competing interests.
1. Schmieder, R. E. Hypertension: How should data from SYMPLICITY HTN-3 be interpreted? Nat. Rev. Cardiol. 11, 375-376 (2014).

2. Bhatt, D. L. et al. A controlled trial of renal denervation for resistant hypertension. N. Engl. J. Med. 370, 1393-1401 (2014).

3. Fadl Elmula, F. E. et al. Renal sympathetic denervation in patients with treatment-resistant hypertension after witnessed intake of medication before qualifying ambulatory blood pressure. Hypertension 62, 526-532 (2013).

4. Hart, E. C. et al. Translational examination of changes in baroreflex function after renal denervation in hypertensive rats and humans. Hypertension 62, 533-541 (2013).

5. Brinkmann, J. et al. Catheter-based renal nerve ablation and centrally generated sympathetic activity in difficult-to-control hypertensive patients: prospective case series. Hypertension 60, 1485-1490 (2012).

6. Fadl Elmula, F. E. et al. Adjusted drug treatment is superior to renal sympathetic denervation in patients with true treatment-resistant hypertension. Hypertension 63, 991-999 (2014).

7. Ormiston, J. A. et al. Renal denervation for resistant hypertension using an irrigated radiofrequency balloon: 12-month results from the Renal Hypertension Ablation System (RHAS) trial. Eurolntervention 9, 70-74 (2013).

8. Ziegler, A. K. et al. Efficacy and safety of renal denervation in elderly patients with resistant hypertension. Catheter Cardiovasc. Interv. http://dx.doi.org/10.1002/ccd.25166.

9. Hering, D. et al. Renal denervation in moderate to severe CKD. J. Am. Soc. Nephrol. 23, 1250-1257 (2012).

10. Zuern, C. S. et al. Effects of renal sympathetic denervation on 24-hour blood pressure variability. Front. Physiol. 3, 134 (2012).

11. Witkowski, A. et al. Effects of renal sympathetic denervation on blood pressure, sleep apnea course, and glycemic control in patients with resistant hypertension and sleep apnea. Hypertension 58, 559-565 (2011).

12. Vase, H. et al. Catheter-based renal denervation for treatment of resistant hypertension. Dan. Med. J. 59, A4439 (2012).

13. Ezzahti, M. et al. Blood pressure and neurohormonal responses to renal nerve ablation in treatment-resistant hypertension. J. Hypertens. 32, 135-141 (2014).

14. Krum, H. et al. Catheter-based renal sympathetic denervation for resistant hypertension: a multicentre safety and proof-of-principle cohort study. Lancet $\mathbf{3 7 3}$, 1275-1281 (2009).

15. Esler, M. D. et al. Renal sympathetic denervation in patients with treatment-resistant hypertension (the Symplicity HTN-2 trial): a randomised controlled trial. Lancet $\mathbf{3 7 6}$, 1903-1909 (2010). 


\section{CORRESPONDENCE}

16. Patel, H. C., Hayward, C. \& Di Mario, C. SYMPLICITY HTN 3: the death knell for renal denervation in hypertension? Glob. Cardiol. Sci. Pract. 2014, 94-98 (2014).

17. Wang, Y. More research is needed to investigate the effect of denervation on blood pressure. Hypertension 63, e85 (2014).

18. Blankestijn, P. J., Bots, M. L., Spiering, W., Leiner, T. \& Voskuil, M. Pro: Sympathetic renal denervation in hypertension and in chronic kidney disease. Nephrol. Dial. Transplant. 29, 1120-1123 (2014)

19. Schlaich, M. P., Sobotka, P. A., Krum, H., Lambert, E. \& Esler, M. D. Renal sympatheticnerve ablation for uncontrolled hypertension. N. Engl. J. Med. 361, 932-934 (2009).

20. Tsioufis, C. et al. Drug-resistant hypertensive patients responding to multielectrode renal denervation exhibit improved heart rate dynamics and reduced arrhythmia burden. J. Hum. Hypertens. 28, 587-593 (2014).

21. Chinushi, M. et al. Blood pressure and autonomic responses to electrical stimulation of the renal arterial nerves before and after ablation of the renal artery. Hypertension 61, 450-456 (2013). 